\title{
Plongée dans le microcosme des cabinets médicaux
}

Felix Schürch

Correspondance: Dr Felix Schürch Altstetterstrasse 118 CH-8048 Zurich

felix.schurch[at]hin.ch
Le voyageur qui prévoit un séjour à l'étranger ira chercher un guide à la bibliothèque tandis que l'assistant qui entame un stage de formation postgraduée recevra, du chef de clinique, le «livre bleu» contenant toutes les directives importantes. Or il n'existait jusqu'ici aucun document de ce type pour les jeunes souhaitant devenir assistant ou assistante médical/e. C'est désormais chose faite avec ce nouveau manuel qui leur permettra de se familiariser avec les tâches complexes d'un cabinet médical. L'auteur, le Dr Emil Schalch, est un médecin de campagne spécialisé dans les questions relatives à la formation des assistantes médicales puisqu'il siège dans différents groupes de travail qui traitent de ce sujet.

Ce manuel de formation bien structuré comprend 32 chapitres, classés en 5 parties. La première partie aborde les questions générales du cabinet médical comme lieu de formation. Différents sujets y sont traités: le secret médical, la manière de gérer les patients, le cadre légal, et bien d'autres encore. La partie consacrée aux tâches administratives traite de l'accueil des patients, des entretiens téléphoniques et des ordonnances. Le chapitre sur le système de facturation permettra à la personne en formation de se familiariser avec le code tessinois, le TARMED et le système du tiers payant, alors que celui consacré au patient traite de questions pratiques allant de la prise de sang au pansement des plaies. Dans la dernière partie, qui porte sur le diagnostic au moyen d'appareils, l'auteur aborde de manière précise et pratique les analyses de laboratoire, les électrocardiogrammes, la fonction pulmonaire et les examens radiologiques.

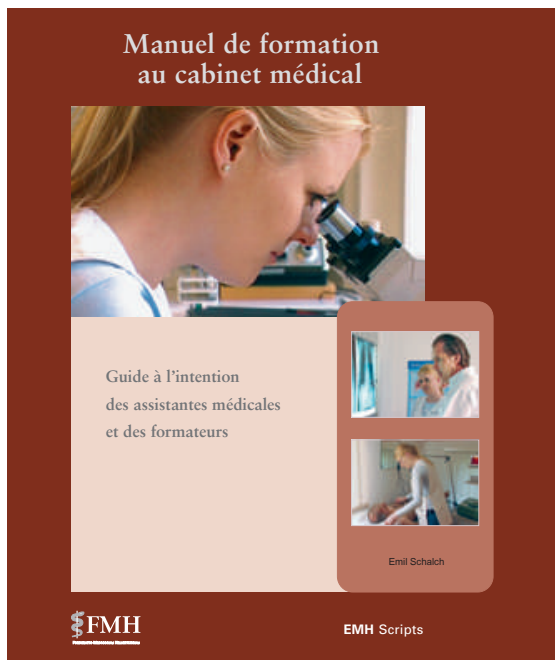

Emil Schalch (auteur)

Fédération des médecins suisses (FMH) (éd.) Manuel de formation au cabinet médical

Guide à l'intention des assistantes médicales et des formateurs

Muttenz: Editions médicales suisses (EMH);

2012.

404 pages. Classeur, 98 CHF.

ISBN 978-3-03754-063-3
Les explications succinctes de l'auteur sont compréhensibles, faciles à lire et parfaitement adaptées au public-cible. Les différents contenus sont présentés sous forme de tableaux et illustrés à l'aide de graphiques. Bien que l'auteur se concentre sur les questions spécifiques, le lecteur remarquera que c'est un médecin et un responsable d'équipe engagé, comme le prouve cette phrase remarquable tirée du chapitre sur le système de facturation: «Arriver à faire le grand écart entre vouloir aider ses concitoyens et réussir dans les affaires est un défi de taille.»

Je trouve ce manuel très utile pour les personnes en formation, car l'auteur y présente les choses de manière concrète et détaillée, sans se dérober derrière des recommandations ou des remarques générales. Pour l'examen de la fonction pulmonaire par ex., il présente un appareil diagnostic spécifique, et pour les examens de suivi recommandés, il récapitule les principaux examens préventifs dans un tableau. Quant au chapitre traitant de l'infrastructure électronique du cabinet, il détaille les étapes de la sauvegarde des données. Dans ce genre d'ouvrage, il est bien sûr inévitable que certains chapitres soient rapidement dépassés et nécessitent des adaptations. C'est pourquoi ce manuel ne se présente pas sous forme de livre mais de classeur, ce qui permet à la personne en formation de remplacer au fur et à mesure les fiches qui auront été modifiées. Un registre par mots-clés clôt ce manuel de plus de 400 pages et en fait un excellent ouvrage de référence.

Ce manuel de formation au cabinet médical doit donc également être recommandé à titre de «guide de voyage» pour les jeunes médecins qui s'installent, tandis que pour les «vieux routards» en pratique privée, il pourra servir de liste de contrôle et leur permettre de repenser les procédures de travail du cabinet ou de trouver de nouvelles idées. Ce manuel s'adresse en premier lieu aux personnes en formation mais aussi aux formateurs et aux détenteurs de cabinets et il ne fait aucun doute qu'il deviendra bientôt leur ouvrage de référence.

\section{Neuerungen in der deutschen Ausgabe des Praxisleitfadens MPA}

Die Angaben zu nützlichen Internet- und Postadressen in Anhang B der deutschen Ausgabe des Praxisleitfadens wurden überarbeitet und sind neu im Internet zugänglich: www.emh.ch $\rightarrow$ bei Suche Bücher «Praxisleitfaden» eingeben $\rightarrow$ deutsche Version anklicken $\rightarrow$ PDF Downloads $\rightarrow$ MPA_Adressen.pdf 